Cahiers de philosophie de l'université de

\title{
Qu'est-ce que la métaphysique ? Une réflexion à partir de l'œuvre de Thomas Nagel
}

\section{Olivier Waymel}

\section{(2) OpenEdition}

1 Journals

Édition électronique

URL : https://journals.openedition.org/cpuc/302

DOI : $10.4000 /$ cpuc.302

ISSN : 2677-6529

Éditeur

Presses universitaires de Caen

\section{Édition imprimée}

Date de publication : 1 novembre 2018

Pagination : 163-186

ISBN : 978-2-84133-904-4

ISSN : 1282-6545

\section{Référence électronique}

Olivier Waymel, « Qu'est-ce que la métaphysique ? Une réflexion à partir de l'œuvre de Thomas Nagel », Cahiers de philosophie de l'université de Caen [En ligne], 55 | 2018, mis en ligne le 01 novembre 2019, consulté le 04 février 2023. URL : http://journals.openedition.org/cpuc/302 ; DOI : https:// doi.org/10.4000/cpuc.302

\section{(c) (7) (8)}

Creative Commons - Attribution - Pas d'Utilisation Commerciale 4.0 International - CC BY-NC 4.0 https://creativecommons.org/licenses/by-nc/4.0/ 


\section{Qu'est-ce que la métaphysique? Une réflexion à partir de l'œuvre de Thomas Nagel}

\section{L'unité de certains problèmes philosophiques importants}

Dans son essai «The Subjective and the Objective», puis dans The View from Nowhere, Nagel développe une analyse de certains problèmes philosophiques fondamentaux: la possibilité de l'esprit dans la nature, la possibilité de la connaissance ou encore la réalité de la liberté.

Selon Nagel, le problème de la possibilité de l'esprit dans la nature ${ }^{1}$ consiste dans le fait que nous sommes spontanément portés à accepter deux affirmations dont la compatibilité n'est pas assurée. D'un côté, je me perçois moi-même essentiellement comme une conscience, et la croyance en l'existence de ma conscience est même la mieux établie de mes croyances. D'un autre côté, les objets de ma conscience semblent être des réalités indépendantes, dont je n'ai conscience qu'à travers leur action sur mon corps. Ma conscience semble donc être seulement un point de vue sur la réalité, et celui-ci peut être comme tel partiel et déformant. Nous sommes par conséquent portés à chercher ce que les choses sont en elles-mêmes. Mais cette recherche d'objectivité semble nous conduire à un tableau de la réalité, que Nagel qualifie de «naturaliste ${ }^{2}$, dont la conscience est absente. Nous n'y trouvons que des corps, car pour expliquer le contenu de notre expérience, il nous suffit de postuler des entités physiques. L'existence de la conscience et sa relation étroite à la nature sont manifestes, mais le tableau naturaliste les laisse inexpliquées. L'état interne et la position de

1. T. Nagel, The View from Nowhere, Oxford, Oxford University Press, 1986, partie III, p. 28-53; trad. fr.: Le point de vue de nulle part, S. Kronlund (trad.), Combas, Éditions de l'éclat, 1993, p. 37-66.

2. T. Nagel, The View..., p. 88; Le point de vue..., p. 106. 
mon corps semblent déterminer mes états conscients, mais comment cette détermination a-t-elle lieu? Comment est-elle même seulement possible? La question des autres esprits manifeste très clairement le problème: nous pensons spontanément que certaines des réalités extérieures dont nous avons conscience sont elles-mêmes conscientes et que leurs états sont systématiquement corrélés aux états physiques de leur corps, c'est-à-dire qu'il y a, pour reprendre l'expression de Nagel, un " effet que cela fait " ${ }^{3} \mathrm{~d}^{\text {'être }}$ ces corps physiques. Cependant, aussi complète que soit notre connaissance de ces corps, nous ne pouvons pas, sur cette seule base, déterminer les états conscients qui leur sont associés, ni même assurer que de tels états leur sont associés.

Considérons le problème de la possibilité de la connaissance empirique ${ }^{4}$. Ici encore, nous sommes spontanément portés à accepter deux affirmations contradictoires. D’un côté, il nous semble que notre expérience nous met en relation directe avec la réalité et nous fournit des connaissances parfois sûres: ainsi, «vous ne doutez pas de l'existence du sol sous vos pieds ou de l'arbre derrière la fenêtre ou de l'existence de vos propres dents ${ }^{5}$. Cette assurance vient de ce que nous n'avons pas conscience des états mentaux qui nous permettent d'être conscients de la réalité, si bien que nous pensons faire l'expérience de la réalité elle-même, et non de son image ${ }^{6}$. Il en résulte que la connaissance paraît aisément accessible. Cependant, nous sommes également spontanément portés à accepter des croyances qui nous conduisent à douter de notre capacité à accéder à la connaissance empirique. Comme nous l'avons vu en exposant le problème de la relation entre le corps et l'esprit, il nous est naturel de nous considérer comme des réalités particulières à l'intérieur du monde, de regarder nos états mentaux comme des propriétés de notre être causées par les autres réalités, et de tenir l'objet immédiat de notre expérience pour un objet distinct de la réalité extérieure. Si nous atteignons dans notre expérience une réalité extérieure, ce ne saurait être que de manière indirecte:

Quelle que soit votre croyance - qu'elle porte sur le soleil, la lune, les étoiles, la maison et le voisinage dans lequel vous vivez, l'histoire, la science, les autres personnes, et même l'existence de votre propre corps, cela est fondé sur vos

3. Voir «What Is It Like to Be a Bat?», repris dans Mortal Questions, Cambridge - Londres New York, Cambridge University Press, 1979, chap. 12, p. 165-180; trad. fr.: Questions mortelles, P. Engel, C. Tiercelin (trad.), Paris, PUF, 1983, p. 193-208.

4. T. Nagel, The View..., partie V, p. 67-89; Le point de vue..., p. 82-108.

5. T. Nagel, What Does It All Mean? A Very Short Introduction to Philosophy, Oxford, Oxford University Press, 1987, p. 7 ; trad. fr.: Qu'est-ce que tout cela veut dire?, R. Ogien (trad.), Combas, Éditions de l'éclat, 1993, p. 11.

6. Ibid. 
propres expériences et pensées, sentiments et impressions sensibles. C’est tout ce dont vous disposez directement, que vous regardiez le livre que vous avez entre les mains, sentiez le sol sous vos pieds, ou vous souveniez que George Washington fut le premier Président des États-Unis, ou que l'eau est $\mathrm{H}^{2} \mathrm{O}$. Tout est plus loin de vous que vos expériences internes et vos pensées, et ne peut être atteint qu'à travers elles?

Notre pensée à propos du monde extérieur est déterminée par deux facteurs: l'action causale de la réalité extérieure et les traits physiques de notre corps, dont notre expérience dépend de manière directe. $\mathrm{Ce}$ second facteur introduit la possibilité d'une déformation. Puisque nous ne connaissons pas, du moins immédiatement, la contribution de ces deux facteurs au contenu de notre expérience, nous ne savons pas dans quelle mesure notre expérience reflète adéquatement la réalité extérieure. Pour disposer d'authentiques connaissances empiriques, il faudrait que nous établissions la fiabilité et la transparence de notre expérience et de nos états mentaux, ou que nous sachions comment notre expérience déforme la réalité, afin d'y apporter les corrections nécessaires. Nous resterons sinon condamnés au scepticisme. Le problème est d'autant plus aigu que les arguments sceptiques sont fondés sur des croyances aussi fortes que celles qu'ils remettent en cause.

Considérons enfin le problème de la liberté. D’un côté, nous trouvons parmi nos croyances les plus solides la croyance que nous sommes des êtres capables d'action : certains événements qui ont lieu dans le monde sont des actions accomplies par un moi, et non de simples événements découlant du devenir réglé de la nature. D’un autre côté, cependant, nos actions présentent parfois certains traits nous conduisant à les considérer elles-mêmes comme les produits d'enchaînements causaux, sociaux, biologiques, etc., et donc comme de simples événements. Or, si nous y regardons de plus près, il semble que toutes nos actions présentent ces traits, et que nous devions par conséquent toutes les considérer comme de simples événements. L'individu destructeur, par exemple, apparaît à la réflexion comme une partie destructrice du monde et comme un être qui est seulement méchant, et non nuisible. Et même si nous rejetons le déterminisme, nous ne voyons pas encore comment un événement, même s'il n'est lié à aucune cause antécédente, pourrait être un événement accompli par moi et constituer une action libre: ne s'agit-il pas simplement d'un événement qui survient d'abord à travers moi, et qui est suivi d'effets hors de moi?

7. Ibid. 
Nos conceptions spontanées de la réalité renferment ainsi un certain nombre de contradictions, ou du moins contiennent des affirmations pouvant rapidement aboutir à des contradictions. Ces contradictions sont ordinairement considérées indépendamment les unes des autres. L'une des thèses fondamentales de Nagel consiste à soutenir que ces contradictions présentent une unité profonde, et l'un des intérêts majeurs de sa pensée réside dans l'analyse qu'il en propose:

Ces questions comportent une structure commune qui nous justifie à localiser une difficulté philosophique commune à toutes, cachée par leur caractère divers $[\ldots]^{8}$.

Les différentes thèses impliquées dans les problèmes que nous avons décrits peuvent être regroupées en deux ensembles.

D'un côté, nous trouvons l'ensemble suivant:

1. à propos de la relation corps-esprit: je suis une conscience; cette conscience est liée à une des réalités physiques particulières qui composent le monde;

2. à propos de la connaissance empirique: notre expérience nous relie directement à un monde extérieur indépendant de nous; elle n'est pas un rêve; nous ne sommes pas des cerveaux dans une cuve, etc.;

3. à propos de la liberté: nous disposons d'un pouvoir d'agir, c'est-à-dire de produire des événements que nous déterminons nous-mêmes et dont nous sommes les auteurs.

D’un autre côté, nous trouvons l'ensemble suivant:

1. à propos de la relation corps-esprit: nos concepts d'états mentaux ont pour contenu des comportements; attribuer un état mental à un organisme, c'est lui attribuer une certaine manière de se comporter dans un certain contexte. Il n'existe dans le monde rien de tel que la conscience;

2. à propos de la connaissance empirique: le contenu de notre expérience semble consister en une simple image de la réalité, et nous ne pouvons par conséquent pas savoir que nous ne sommes pas des cerveaux dans une cuve, ou que nous ne rêvons pas, et plus généralement que nos états de conscience représentent la réalité extérieure de manière adéquate;

8. T. Nagel, Mortal Questions, chap. 14, «Subjective and Objective», p. 206; Questions mortelles, p. 237. 
3. à propos de la liberté: nos actions ne sont que les effets de ce que la nature, notre éducation et notre environnement ont fait de nous, et nous ne sommes en vérité les auteurs d'aucun événement dans le monde.

Les thèses du premier ensemble ont pour point commun qu'elles s'imposeraient à nous sans réserve si nous considérions le monde uniquement tel qu'il se donne immédiatement à notre conscience et laissions de côté notre croyance que cette conscience est une propriété d'un être qui se trouve dans le monde et appartient à la nature que notre conscience prétend atteindre. Selon cette perspective, le monde se limite à l'objet immédiat de notre conscience, et il ne nous contient pas en tant que sujets. Nous considérons ici le monde de l'extérieur, comme si notre moi n'y appartenait pas. L'unité des trois thèses en question consiste donc en ce qu'elles relèvent d'un point de vue interne sur la réalité.

Les trois thèses du second ensemble ont en commun de relever d'une autre perspective. Elles s'imposent lorsque nous cessons de considérer le monde depuis ce point de vue interne, depuis lequel nous ne nous apparaissons pas à nous-mêmes, et considérons notre pensée et notre expérience comme des propriétés de réalités naturelles, et plus précisément comme des images de la réalité extérieure agissant causalement sur nous. Lorsque nous adoptons ce point de vue externe, nous développons une conception nouvelle de la réalité, que Nagel appelle le «tableau naturaliste», dont nous prétendons qu'elle représente ce que la réalité est en elle-même. Les trois thèses du second ensemble font partie de ce tableau. Premièrement, de ce point de vue, la conscience est introuvable: nous ne nous apparaissons plus comme des consciences, mais comme de simples organismes physiques. Deuxièmement, le scepticisme empirique apparait inévitable: si nos croyances à propos des choses extérieures résultent des effets physiques que ces choses produisent sur notre corps, et non d'une expérience directe de ces choses, leur adéquation est sujette au doute. Troisièmement, le libre arbitre apparait impossible, que le déterminisme soit vrai ou non: s'il est vrai, nos actions apparaissent comme les conséquences des facteurs naturels et culturels qui nous ont formés, et non de nous, et nous n'en sommes pas les auteurs; s'il n'est pas vrai, elles apparaissent tout de même dépendre de motifs, désirs ou valeurs qu'il ne dépend pas de nous de suivre, et nous ne pouvons toujours pas nous considérer comme leurs auteurs.

Ainsi, si nous suivons l'analyse que propose Nagel, un certain nombre de problèmes philosophiques parmi les plus importants de la tradition résultent de notre capacité à adopter deux points de vue contradictoires sur nous-mêmes et sur le monde. Selon nous, la compréhension de l'origine et de l'unité de ces problèmes peut contribuer, outre son intérêt propre, 
à atteindre trois objectifs importants: comprendre leur place à l'intérieur de l'enquête philosophique, saisir la nature de celle-ci, et plus précisément la nature de la réflexion métaphysique, et enfin développer une méthode adéquate pour l'enquête métaphysique, ou du moins pour une part de celle-ci.

\section{La réflexion métaphysique comme recherche d'une conception externe}

Essayons d'abord de montrer que l'idée de point de vue externe est centrale pour la pensée métaphysique, du moins pour la pensée métaphysique moderne, et que la métaphysique doit être considérée comme la recherche d'une conception externe satisfaisante. Comme on le sait, la métaphysique comporte traditionnellement deux types d'enquêtes ${ }^{9}$. Elle est d'une part ontologie, description de ce en quoi peut consister le fait d'être. Elle cherche alors à répondre à des questions du type: "les possibles sont-ils réels?", "peut-on prouver l'existence de Dieu?», «qu'est-ce qu'une propriété?», etc. Elle est d'autre part réflexion sur ce qui est, plus précisément sur la nature de ce qui existe effectivement dans notre monde, et notamment sur la possibilité qu'il existe dans notre monde autre chose que le contenu immédiatement présent dans notre expérience. Par ce second aspect, la métaphysique s'interroge sur l'existence de réalités n'appartenant pas à l'ensemble des objets immédiats de notre expérience. Les objets de ces deux enquêtes sont distincts. Il serait par conséquent sans doute conforme à la nature des choses de les désigner par deux termes différents. Pour cela, nous disposons de trois termes: la métaphysique, l'ontologie, la théologie. Aristote désigne les deux types d'enquêtes en question par les termes d'ontologie et de théologie. Mais le terme de théologie ne convient pas pour désigner l'enquête sur l'étendue de ce qui est, et notamment sur ce qui est au-delà du contenu immédiat de notre expérience, la théologie n'étant qu'une part de cette enquête, celle qui porte sur Dieu. Nous suggérons de désigner par le terme d'ontologie l'investigation sur l'être en tant qu'être, et par celui de métaphysique l'investigation sur les réalités qui ne constituent pas le contenu immanent de notre expérience. Nous distinguons donc la métaphysique comprise au sens large, aristotélicien, qui englobe également l'ontologie, et la métaphysique comprise au sens étroit, qui pose exclusivement la question de l'existence et de la nature des réalités transcendant le contenu de l'expérience immanente.

9. Voir P. Aubenque, Le problème de l'être chez Aristote, Paris, PUF, 1962. 
Or, la question centrale de la métaphysique comprise au sens étroit, «qu'y a-t-il en dehors du contenu immédiat de notre expérience?», se détermine, pour nous modernes, comme la question de l'élaboration d'un point de vue externe. En effet, depuis le développement des conceptions modernes de la perception, il semble que notre conception spontanée de la réalité doive être tenue pour une image d'une réalité distincte d'elle, car elle semble élaborée à partir des effets physiques que des choses extérieures produisent sur nos organes des sens ${ }^{10}$. Notre expérience nous apparaît comme une propriété d'un être particulier dans le monde, âme ou corps, et non comme la simple présence de la réalité. La recherche de la vérité doit alors prendre la forme d'une enquête métaphysique à propos de la nature de la réalité dont notre expérience n'est semble-t-il qu'une image peut-être partielle et déformée, c'est-à-dire la forme d'une recherche de ce que Nagel appelle une "conception externe» de la réalité ${ }^{11}$. Nous allons maintenant essayer de décrire les questions que rencontre l'enquête métaphysique comprise comme recherche d'une conception externe.

\section{Le premier moment de l'enquête métaphysique: y a-t-il une réalité transcendante au-delà du donné immanent de l'expérience?}

La métaphysique semble donc consister dans la recherche d'une conception externe satisfaisante. Cette question ne vient cependant en vérité qu'en second lieu. Une question la précède, portant sur la simple possibilité d'adopter un point de vue externe sur nous-mêmes et sur l'intelligibilité d'un tel point de vue.

Il semble impossible de rendre compte de certains aspects de notre expérience si nous ne postulons pas l'existence de réalités se trouvant hors de notre expérience et causant le contenu de celle-ci et si nous ne regardons pas notre expérience comme une image de la réalité, et non comme la simple présence de celle-ci à notre esprit. Nous ne pouvons cependant pas suivre aveuglément cette intuition. La croyance qu'il existe hors de nous des réalités autres que notre corps, distinctes de la conscience que nous pouvons en avoir, ainsi que la croyance que la recherche d'une conception absolue a un sens, doivent être examinées de manière systématique. Cet examen relève de la métaphysique. Il correspond plus précisément à la première question métaphysique: demander s'il existe autre chose que ce

10. Voir P. Hamou, Voir et connaître à l'âge classique, Paris, PUF, 2002.

11. T. Nagel, The View..., partie II, chap. 3, p. 21; Le point de vue..., p. 28. 
qui est donné dans l'expérience, autre chose que le contenu immanent de l'expérience, et même si cette question a un sens, ce n'est pas autre chose que de demander si la métaphysique prise au sens étroit a un objet. La première question métaphysique est donc celle-ci: le point de vue interne est-il le seul point de vue sur la réalité? Autrement dit, est-ce que, depuis ce point de vue, c'est la réalité elle-même qui nous est donnée? Ou devons-nous considérer, comme nous y sommes spontanément portés, qu'un autre point de vue, externe, est concevable? Cette question ne porte pas encore sur la nature des réalités transcendantes, qui sont les objets propres de l'enquête métaphysique. Elle porte sur la réalité de ce domaine et par conséquent sur la réalité des questions métaphysiques s'enquérant de la nature des réalités transcendantes.

Un courant de la philosophie, couvrant notamment la phénoménologie et la pensée de Wittgenstein, a nié que cette question ait un sens. Nous ne nous y arrêterons pas. Ce n'est cependant que si nous rejetons ses raisons que l'enquête métaphysique, comprise au sens large, aristotélicien, possède un objet propre et ne peut être réduite à l'ontologie. La question est alors la suivante: en quoi la conception externe de la réalité consiste-t-elle?

\section{Le deuxième moment de l'enquête métaphysique: la recherche d'une conception externe satisfaisante}

\section{La méthode de l'objectivité}

Comment pouvons-nous nous approcher de la «conception absolue de la réalité»? La difficulté semble insurmontable: comment pourrions-nous déterminer le contenu du point de vue externe si nous ne pouvons sortir de nous-mêmes et donc adopter ce point de vue? À l'idée de cette conception absolue ne se trouve en effet associée pour nous aucune expérience. Il semble que nous ne puissions faire davantage que concevoir l'idée de ce point de vue. Il nous est cependant possible, par ce que Nagel appelle la «méthode de l'objectivité ${ }^{12}$, de nous approcher de la conception absolue de la réalité. Cette méthode s'élabore de la manière suivante:

La première étape consiste à voir que nos perceptions sont causées par l'action des choses sur nous, à travers leurs effets sur nos corps, qui sont eux-mêmes des parties du monde physique ${ }^{13}$.

12. Nagel décrit cette méthode dans The View..., partie II, chap. 1, p. 13-17.

13. T. Nagel, The View..., partie II, chap. 1, p. 14; Le point de vue..., p. 20. 
Or, si nous sommes des réalités parmi d'autres dans le monde et si les réalités composant le monde entretiennent les unes avec les autres des relations causales, notre perception elle-même résulte de relations causales. Notre expérience est donc seulement une image ou une représentation de la réalité:

L'étape suivante consiste à réaliser que, puisque les mêmes propriétés physiques qui causent des perceptions en nous par l'intermédiaire de nos corps produisent aussi des effets différents sur les autres choses physiques et peuvent exister sans causer aucune perception, leur véritable nature peut être détachée de leur apparence perceptuelle et ne lui ressemble pas nécessairement ${ }^{14}$.

La direction dans laquelle nous devons nous engager pour nous approcher de la conception absolue apparaît alors. Il s'agit, et c'est là la troisième étape dans l'élaboration de la méthode de l'objectivité,

[d']essayer de former une conception de la vraie nature [des choses], qui est indépendante à la fois de son apparence pour nous et de son apparence pour les autres êtres capables de perception. Cela signifie non seulement ne pas penser au monde physique depuis notre propre point de vue particulier, mais ne pas y penser non plus depuis un point de vue perceptuel humain plus général $[\ldots]^{15}$.

Essayons de déterminer plus précisément cette méthode. Les croyances que nous développons depuis notre point de vue interne sont déterminées à la fois par ce que nous sommes et par la réalité extérieure, comme les images sont déterminées par leur support et par les objets qu'elles représentent. Des facteurs internes, biologiques, culturels et historiques, contribuent à l'image que nous nous faisons de la réalité. Pour découvrir ce qu'est en elle-même la réalité que nous visons dans notre expérience, nous pouvons, à défaut de pouvoir sortir de nous-mêmes, essayer d'éliminer de notre conception les aspects dont la source se trouve en nous.

Le progrès en direction de la conception absolue repose plus précisément sur deux principes. Premièrement, si le contenu de notre expérience est causé par des réalités extérieures indépendantes, alors, puisqu'il n'y a pas de différence dans les effets sans différence dans les causes, ceux de ses aspects qui sont causés par l'action des choses extérieures, et non par les propriétés de notre corps, reflètent les relations qu'entretiennent les choses elles-mêmes. Nous pouvons par conséquent tirer des relations entre nos expériences une connaissance des relations entre les éléments de la réalité,

14. Ibid.

15. Ibid. 
ou du moins une connaissance partielle de celle-ci, tous les aspects de la réalité n'exerçant pas une action causale sur notre expérience. L'examen de notre expérience nous permet de connaître les relations qu'entretiennent entre elles les composantes de la réalité exerçant un pouvoir causal sur notre expérience. Cet examen ne nous permet cependant d'accéder qu'à une connaissance exclusivement formelle de la réalité extérieure.

Un deuxième principe permet de développer notre saisie de la conception absolue de la réalité à partir du point de vue interne que nous avons sur elle. Ce principe nous autorise, dans certaines conditions, à attribuer à la réalité externe certains des aspects qualitatifs, et non plus seulement relationnels, que présente notre expérience ${ }^{16}$. Comme propriétés d'êtres particuliers, nos expériences doivent pouvoir être expliquées, comme toute chose. Pourquoi, pouvons-nous par exemple demander, sommes-nous en train de percevoir une forme carrée rouge? Afin de déterminer ce qu'est la réalité en elle-même, nous pouvons chercher ce que doit être cette réalité pour qu'elle nous apparaisse comme elle le fait. Nous pouvons espérer être alors en mesure de séparer à l'intérieur de notre expérience ce qui est déterminé par la réalité extérieure et ce qui est déterminé par nos propres caractères. Ce n'est par exemple pas en attribuant aux choses extérieures les qualités sonores et colorées sous lesquelles elles apparaissent à notre point de vue interne que nous pouvons le mieux expliquer nos expériences et leurs variations. Celles-ci peuvent être mieux expliquées si nous considérons nos expériences des sons et couleurs comme des qualités secondes, c'està-dire comme des propriétés de nous-mêmes, images ou représentations subjectives de certaines propriétés des choses qui sont d'une autre nature et qui doivent être décrites en d'autres termes, en l'occurrence en termes d'étendue et de mouvement. En éliminant les qualités secondes de notre conception de la réalité et en leur substituant des qualités premières, nous parvenons à une conception plus objective de la réalité, moins dépendante de nos propres déterminations biologiques. Nous ne concevons alors plus le monde physique tel qu'il se présente depuis le point de vue particulier que nous occupons, ni même depuis le point de vue perceptuel plus général qui est celui de l'homme. Cependant, dira-t-on, avons-nous le droit d'attribuer aux choses telles qu'elles sont les qualités premières par lesquelles nous expliquons les divers contenus que prend notre expérience? Ne peut-il pas de nouveau s'agir d'une simple apparence sous laquelle la réalité nous apparaît? Il est certes possible qu'il en soit ainsi, mais nous n'avons aucune raison de le penser, et c'est en attribuant aux choses elles-mêmes exclusivement

16. T. Nagel, The View..., partie VI, chap. 3, "Kant and Strawson », p. 99-105; Le point de vue..., p. 119-126. 
des qualités premières que nous pouvons le mieux expliquer la forme que prennent nos expériences. Cela suffit à justifier que nous attribuions ces qualités à la réalité telle qu'elle est en elle-même, même si cette justification n'est pas une démonstration. Nous pouvons donc non seulement attribuer aux composants de la réalité les relations que nous trouvons dans notre expérience de la réalité, mais encore leur attribuer un certain type de qualités, les qualités premières.

Cette méthode de l'objectivité consistant à éliminer de notre conception du monde tous les éléments dépendant de ce que nous sommes, nous sujets de cette conception, constitue une alternative à l'impossible sortie de soi. Elle nous conduit, tout en restant à l'intérieur de notre pensée comme pensée d'un être particulier appartenant au monde, à substituer des conceptions plus objectives à nos conceptions subjectives spontanées. Nous parvenons ainsi à élaborer une conception substantielle de la réalité extérieure que nous avons le droit de considérer comme vraie, ou plus proche de la conception absolue de la réalité. Cette conception de la réalité à laquelle nous parvenons par l'application de la méthode de l'objectivité constitue ce que Nagel appelle le "tableau naturaliste ${ }^{17}$ de la réalité, ou du moins, comme nous le verrons, une partie de celui-ci.

En quoi ce tableau consiste-t-il? Ce tableau découle de la recherche d'une explication du contenu de nos expériences. Ce travail est accompli par les sciences de la nature. En effet, celles-ci, en cherchant à expliquer nos expériences, sont conduites à postuler des entités théoriques. Or, si nous acceptons la légitimité du point de vue externe, il est rationnel de regarder les entités théoriques des sciences de la nature comme des hypothèses métaphysiques, et la science comme une enquête permettant d'atteindre le but visé par la réflexion métaphysique, la connaissance des réalités transcendantes. Dans ce moment de son enquête, le métaphysicien ne fait pour l'essentiel qu'affirmer la réalité de ces entités théoriques.

\section{Les limites de la méthode de l'objectivité}

La réflexion métaphysique semble cependant déborder la simple projection métaphysique des résultats de la science: les considérations sur l'existence de Dieu ou sur l'existence de principes téléologiques sont extérieures aux sciences de la nature, mais elles font manifestement partie de la recherche d'un tableau adéquat de ce que la réalité est en elle-même. Cependant, les succès de la méthode de l'objectivité ont conduit nombre de philosophes à considérer

17. T. Nagel, The View..., partie V, chap. 6, p. 88; Le point de vue..., p. 106. 
que cette méthode constitue la méthode exclusive de la métaphysique. Si l'on accepte ce présupposé, la réalité transcendante ne peut contenir que des entités et des propriétés constituant des causes immédiates du contenu de notre expérience. Elle ne peut contenir que des réalités physiques, les seules que la méthode de l'objectivité peut découvrir. On peut appeler «objectiviste» cette version du naturalisme. Il est possible que l'une ou l'autre des formes du naturalisme objectiviste soit vraie et qu'il n'existe pas d'autres réalités et propriétés que les réalités et les propriétés décrites par la physique. Cela ne peut cependant pas être accepté de manière $a$ priori, même si les succès de la méthode de l'objectivité lui confèrent une autorité considérable. Cela doit être établi à travers une confrontation avec les conceptions métaphysiques concurrentes. L'acceptation a priori de la méthode de l'objectivité comme méthode unique de la métaphysique constitue selon Nagel l'une des formes de l'erreur la plus commune en philosophie, l'idéalisme, qui consiste à soutenir ou à présupposer que la réalité est limitée à ce qui peut être connu par une certaine méthode de connaissance, ou à ce qui peut être connu de manière générale, c'est-à-dire à adopter un "critère épistémologique de la réalité ${ }^{18}$. Pourquoi, demande Nagel, la réalité devrait-elle se borner à ce que nous pouvons en connaître, et plus encore à ce que nous pouvons en connaitre par une certaine méthode? Il est vrai que la réflexion métaphysique parvient à des résultats mieux établis à propos des causes prochaines de notre expérience qu'à propos de Dieu et des principes téléologiques, qui, s'ils se manifestent dans l'expérience, le font de manière plus indirecte encore. Mais le fait qu'un aspect de la réalité ne puisse pas être connu avec certitude ne justifie en rien l'affirmation que cet aspect n'existe pas.

La réflexion métaphysique fondée sur l'examen du contenu de notre expérience ne peut donc pas être réduite $a$ priori à une simple projection métaphysique des résultats des sciences de la nature. Il est possible que la réalité extérieure déborde ce que la méthode de l'objectivité permet d'en découvrir. Cela est possible parce que notre expérience de la réalité n'est pas un point de vue sur celle-ci seulement au sens où elle est une expérience subjective de la réalité, déterminée en partie par les caractères du sujet qui en fait l'expérience, mais aussi au sens où cette expérience est incomplète. La méthode de l'objectivité cherche à atteindre une conception absolue des seules réalités qui nous affectent causalement dans notre expérience de manière directe, mais il est possible qu'il existe des réalités n'exerçant pas une action causale directe sur notre expérience : il est possible qu'il existe un Dieu, que certaines réalités naturelles possèdent des propriétés épiphénoménales, ou

18. T. Nagel, The View..., partie VI, chap. 1, p. 91; Le point de vue..., p. 110. 
que la nature soit gouvernée par des principes téléologiques. Ces aspects de la réalité, s'ils existent, échappent nécessairement à une enquête métaphysique fondée sur la seule méthode de l'objectivité et les seuls résultats des sciences de la nature. Qu'en est-il de cette possibilité? C'est là une question que le métaphysicien doit poser. La recherche métaphysique d'un tableau de ce qu'est en elle-même la réalité extérieure ne peut se borner a priori à une validation de l'image de la réalité qu'élaborent les sciences de la nature. Celles-ci n'ont pas réduit le travail du métaphysicien à opérer une projection dans la réalité en soi des résultats scientifiques.

Le problème est alors de savoir comment nous pouvons découvrir les aspects de la réalité transcendante qui se trouvent peut-être au-delà de la portée de la méthode de l'objectivité. Cette connaissance n'est peutêtre pas complètement au-delà de nos ressources épistémologiques. Il est possible que ces aspects de la réalité déterminent notre expérience de façon indirecte, par exemple à travers l'ordre de succession des phénomènes, ou à travers certaines propriétés du monde réel que ne possèdent pas les autres mondes possibles. Il peut sembler que Nagel s'engage dans une telle recherche dans certains de ses textes les plus récents, par exemple lorsque, dans son essai "Public Education and Intelligent Design ${ }^{19}$, il cherche à montrer que l'hypothèse du dessein intelligent ne doit pas être exclue a priori, mais au contraire considérée comme une hypothèse devant être prise au sérieux et évaluée à travers un examen empirique, de la même façon que l'hypothèse rivale proposée par le néo-darwinisme. Son propos relève cependant en vérité alors plus de l'épistémologie de la métaphysique que de la métaphysique elle-même. Il cherche moins à défendre des thèses métaphysiques non physicalistes déterminées qu'à critiquer l'acceptation a priori, idéaliste, largement répandue, de la thèse selon laquelle la méthode de l'objectivité suffirait à élaborer un tableau complet de la nature et serait ainsi la méthode exclusive de la métaphysique. De la même façon, il peut sembler que dans son dernier ouvrage, Mind and $\operatorname{Cosmos}^{20}$, Nagel présente en faveur du rejet de l'explication matérialiste néo-darwinienne de la vie suggérée par le tableau naturaliste, des raisons positives fondées sur le contenu de l'expérience. Mais ces raisons ne relèvent pas, en vérité, d'une réflexion métaphysique sur le contenu de l'expérience que nous faisons de la réalité objective. Elles découlent de l'impossibilité d'intégrer dans le tableau

19. T. Nagel, «Public Education and Intelligent Design», Philosophy and Public Affairs, t. 36, $\mathrm{n}^{\circ}$ 2, 2008, p. 187-205. Voir aussi «Dawkins and Atheism», Times Literary Supplement, 7 mai 2004; et «Why Is There Anything?», Times Literary Supplement, 7 mai 2007.

20. T. Nagel, Mind and Cosmos: Why the Materialist Neo-Darwinian Conception of Nature Is Almost Certainly False, Oxford, Oxford University Press, 2012. 
naturaliste le fait évident, introduit par le point de vue interne et non par une réflexion sur le contenu de notre expérience, que la conscience existe. La réflexion de Nagel relève ici du troisième type de problèmes métaphysiques, sur lequel nous nous arrêterons dans la prochaine section. Il est toutefois peut-être possible, par l'examen du contenu de l'expérience, de compléter le tableau naturaliste issu de la méthode de l'objectivité. La fragilité des spéculations que la théologie naturelle a pu produire dans l'histoire de la métaphysique, par exemple, montre que cette tâche est difficile. Disons seulement qu'elle ne peut en tous les cas être menée à bien que si l'on s'appuie sur le tableau naturaliste résultant de la méthode de l'objectivité. Il n'est en effet rationnel d'accepter l'une ou l'autre des hypothèses théologique et téléologique que si le cadre fourni par la conception de la nature élaborée par l'application exclusive de la méthode de l'objectivité ne permet pas d'expliquer les phénomènes de manière aussi satisfaisante que cette hypothèse, en dépit de son coût métaphysique. La réflexion métaphysique sur l'existence de principes théologiques et téléologiques est par conséquent elle aussi un prolongement métaphysique de l'enquête scientifique, de même que l'enquête à propos des causes de l'expérience. Il appartient donc aux sciences de la nature d'accomplir l'ensemble du second moment de l'enquête métaphysique et de rechercher, à partir du contenu de notre expérience, une image adéquate de la réalité externe.

\section{Le troisième moment de l'enquête métaphysique: comment résoudre les conflits entre les points de vue interne et externe?}

\section{Le tableau naturaliste en question:}

les contradictions entre les points de vue interne et externe

La métaphysique doit donc en premier lieu poser la question de la réalité du point de vue externe, et en second lieu chercher à élaborer, à partir du contenu immanent de notre expérience, une conception adéquate de la réalité transcendante. L'enquête métaphysique ne trouve cependant pas encore son point final dans cette conception. Celle-ci soulève en effet certaines difficultés: elle entre en contradiction en divers points avec certaines croyances profondément établies du point de vue interne, sans pourtant parvenir d'emblée à nous en imposer le rejet. Ce sont ces points de contradiction que nous avons décrits dans la première section. Les résistances de certaines croyances du point de vue interne face au tableau naturaliste constituent autant de raisons de douter de ce dernier. Le tableau 
naturaliste du point de vue externe ne peut être considéré comme le dernier mot de l'enquête métaphysique s'il ne peut intégrer les croyances les plus fortes du point de vue interne, ou du moins si l'on n'y ajoute pas des raisons suffisantes de tenir le point de vue interne pour illusoire. Et si aucune de ces deux voies ne peut être empruntée avec succès, le tableau naturaliste devra être modifié de telle manière que les contradictions entre les deux points de vue soient surmontées. L'examen des contradictions entre les deux points de vue interne et externe constitue ainsi un aspect essentiel de la réflexion métaphysique, car ces contradictions peuvent nous permettre d'élaborer une conception plus adéquate de ce que la réalité est en elle-même. Il constitue, à côté de la réflexion critique sur l'expérience, et notamment à côté de la méthode de l'objectivité, le second aspect d'une méthode métaphysique rigoureuse.

\section{Tableau a priori des solutions possibles aux conflits des points de vue interne et externe}

En comprenant que certains des problèmes philosophiques les plus importants de la tradition consistent en conflits entre notre point de vue interne spontané sur la réalité et le point de vue externe auquel nous parvenons à nous élever, nous saisissons mieux leur place à l'intérieur de l'enquête métaphysique. Nous nous rendons également capables de décrire l'ensemble fermé des types de solutions qu'il est possible d'y apporter, et de cette façon de rendre plus précise et rigoureuse l'évaluation des arguments avancés en faveur de chacune des positions possibles.

Pour chacun de ces problèmes, le principe qui doit guider la recherche de la solution consiste à rechercher un arbitrage entre les deux points de vue. Comme l'écrit Nagel,

[...] quelque chose doit céder le terrain, car deux façons de penser naturelles et nécessaires en arrivent à se heurter et ne peuvent être accommodées sans certains aménagements en un unique point de vue sur la façon dont les choses sont ${ }^{21}$.

Essayons de décrire les différentes formes que peut prendre la solution.

\section{La solution idéaliste forte}

Une première réponse aux problèmes métaphysiques de contradiction des points de vue est la réponse idéaliste. Elle consiste à rejeter le point de vue

21. T. Nagel, Mortal Questions, chap. 14, p. 210; Questions mortelles, p. 242. 
externe et l'interprétation métaphysique du tableau naturaliste. Selon cette conception, les problèmes métaphysiques de contradiction entre les points de vue ne se posent que si nous considérons qu'il y a une réalité extérieure transcendante agissant causalement sur notre corps et déterminant de cette manière le contenu de notre expérience. En rejetant le point de vue externe, nous rejetons, pour chacun des problèmes en question, l'une des deux affirmations contradictoires, de sorte que les problèmes en question se trouvent résolus, ou plutôt dissous. Comme l'écrit Strawson à propos du problème particulier de la connaissance empirique, cette voie consiste "moins à affronter la difficulté qu'à l'esquiver ${ }^{22}$. Il s'agit d'une réponse extrême aux problèmes métaphysiques, consistant à les considérer comme de faux problèmes, résultant d'une mauvaise interprétation de certains énoncés de nos conceptions ordinaires, plus précisément d'une mécompréhension de ce que sont le point de vue externe et la méthode de l'objectivité. Selon l'idéaliste, les énoncés que l'on attribue au point de vue externe affirment seulement que les données immanentes du point de vue interne présentent un certain ordre. Correctement compris, ils ne contiennent aucune référence à des réalités extérieures au contenu immanent de l'expérience et ne possèdent donc aucune dimension métaphysique. L'idéalisme consiste ainsi à réinterpréter le point de vue externe en réduisant les entités substantielles de la métaphysique à de simples propriétés formelles des phénomènes. Ainsi, par exemple, d'après l'idéalisme, l'esprit conscient n'est pas une propriété qui appartiendrait à certaines réalités naturelles. Il ne s'agit pas d'une propriété naturelle, mais de l'acte d'un sujet transcendantal qui échappe à toute réflexion essayant de le saisir comme un objet dans le monde.

Selon Nagel, cette position est celle qu'adoptent, de deux façons très différentes, d'un côté la phénoménologie de Husserl et d'un autre côté Wittgenstein, aussi bien dans le Tractatus que dans les Recherches philosophiques ${ }^{23}$. C'est Wittgenstein qui, selon Nagel, a emprunté cette voie de la façon la plus influente, et c'est lui qui a contribué de la façon la plus décisive à faire $\mathrm{du} \mathrm{XX}^{\mathrm{e}}$ siècle un siècle philosophiquement idéaliste ${ }^{24}$. On

22. P. F. Strawson, Skepticism and Naturalism, Londres, Routledge, 1987, partie I, chap. 1, p. 3 (nous traduisons).

23. T. Nagel, Mortal Questions, chap. 14, p. 212; Questions mortelles, p. 244. Une solution de ce type est également adoptée par Russell dans Our Knowledge of the External World as a Field for Scientific Method in Philosophy (Londres, G. Allen \& Unwin, 1922) et par Austin dans Sense and Sensibilia (Oxford, Oxford University Press, 1962).

24. Nagel suit ici le jugement de Bernard Williams, selon qui les dernières conceptions de Wittgenstein à propos de la signification sont «une des sources les plus importantes de l'idéalisme contemporain» (T. Nagel, The View..., partie VI, chap. 4, p. 105; Le point de vue..., p. 126). Voir B. Williams, "Wittgenstein and Idealism», repris dans Moral Luck: Philosophical Papers, 1973-1980, Cambridge, Cambridge University Press, 1981, p. 144-163. 
peut considérer que Wittgenstein cherche à montrer que ce que Nagel appelle le point de vue externe constitue une illusion et que le monde se réduit à ce qui est manifesté au point de vue interne. De même, la phénoménologie de Husserl doit être considérée comme une réponse idéaliste aux problèmes métaphysiques de contradiction des points de vue. Husserl s'intéresse plus particulièrement au problème de la possibilité de la connaissance, mais la réponse qu'il propose peut être aisément transposée à l'ensemble des problèmes métaphysiques. Selon Husserl, le défi sceptique, qui est insurmontable, est une conséquence de ce qu'il nomme «l'attitude naturelle», qui consiste à considérer l'expérience comme la propriété d'un être naturel, c'est-à-dire à adopter sur le monde un point de vue externe. Il s'agit là selon Husserl d'une mécompréhension de l'expérience dérivant d'une compréhension de soi et des choses calquée sur le modèle des choses naturelles. En vérité, selon Husserl, les réalités transcendantes sont le produit d'une constitution qui prend pour matière le vécu immanent, c'est-à-dire le contenu de ce que Nagel appelle le point de vue interne, et ces réalités ne sont qu'une forme du donné, et non quelque chose qui en serait ontologiquement indépendant. Husserl et Wittgenstein développent ainsi une pensée anti-métaphysique radicale selon laquelle les problèmes métaphysiques de conflits des points de vue constituent des illusions et se résolvent par la simple analyse de leurs termes.

Passons maintenant à l'examen des autres types de réponses aux différents problèmes métaphysiques de conflits de points de vue. Toutes ont en commun d'accepter la réalité de ces problèmes, et elles doivent pour cette raison être qualifiées de réalistes.

\section{Les solutions idéalistes faibles}

Un second type de réponses consiste à adopter ce qu'on peut appeler un idéalisme faible. On reconnaît ici la possibilité d'adopter un point de vue externe sur nous-mêmes, et donc l'authenticité des problèmes métaphysiques, et on résout les problèmes en question en rejetant comme fausses, et non comme dépourvues de sens, les croyances du point de vue externe qui entrent en contradiction avec des croyances irrésistibles du point de vue interne. Ce type d'idéalisme ne répond pas de manière générale aux problèmes de conflits des points de vue: c'est toujours à tel ou tel conflit entre les deux points de vue qu'il prétend apporter une réponse.

\section{Le naturalisme objectiviste}

Un troisième type de solutions aux problèmes métaphysiques de conflits entre les points de vue interne et externe consiste à considérer que la conception de la réalité élaborée par la méthode de l'objectivité et la 
réflexion sur l'ordre de l'expérience fournit une conception complète de la réalité telle qu'elle est en elle-même, et que les problèmes de conflits de points de vue ne peuvent être résolus à travers une modification du point de vue externe, qu'il s'agisse d'y ajouter ou d'en soustraire un élément. La méthode de l'objectivité et la réflexion sur l'ordre de l'expérience fournissant seules, selon ces conceptions, le contenu du point de vue externe, elles peuvent être regroupées sous le terme de naturalisme objectiviste. Selon cette forme de naturalisme, les aspects de notre conception interne qui ne peuvent pas être intégrés au point de vue externe doivent être considérés comme de simples apparences d'aspects de la réalité dont il nous faut encore rechercher la véritable nature. Et si nous sommes réticents à l'égard des solutions de ce type, c'est uniquement parce que nous ne parvenons pas à cesser de croire en la réalité de ces apparences, si bien que la force de notre intuition ne doit pas être considérée comme la manifestation que la réalité comporte probablement des aspects que le tableau naturaliste n'est parvenu à refléter. Ces réticences reposent sur des illusions.

Ajoutons que le programme du naturalisme objectiviste peut être accompli de deux manières, par réduction ou par élimination, que Nagel décrit ainsi :

[...] la réduction: on peut essayer de sauver les apparences autant que possible, en les accommodant à l'aide d'une interprétation objective. Ainsi [...] on pourrait analyser l'expérience en termes de critères comportementaux, ou l'agir en termes de certaines sortes de causes.

[...] l'élimination: si aucune réduction ne paraît plausible, on peut rejeter comme une illusion la formulation d'un point de vue subjectif, en proposant peut-être une explication de la manière dont il apparaît. Par exemple, on pourrait dire [...] que la liberté d'agir n'existe pas, que les expériences peuvent être caractérisées de manière adéquate par leur rôle causal et qu'elles ne possèdent pas de qualités phénoménologiques en plus ${ }^{25}$.

\section{Les solutions déflationnistes}

L'idéalisme, fort ou faible, et le naturalisme objectiviste ne sont cependant pas les seules réponses possibles aux problèmes métaphysiques de conflits des points de vue, même s'ils dominent notre contexte philosophique et culturel ${ }^{26}$. Il s'agit de solutions extrêmes consistant à adopter complètement l'un des points de vue au détriment de l'autre, dont le succès repose sur la

25. T. Nagel, Mortal Questions, chap. 14, p. 210-211; Questions mortelles, p. 242.

26. T. Nagel, The View..., partie I, p. 5 ; Le point de vue..., p. 9. 
simplicité, plus précisément sur le fait qu'il semble nécessaire de choisir entre la vérité du point de vue interne et la vérité du point de vue externe:

La source profonde de l'idéalisme comme de son opposé objectiviste est la même: une conviction qu'un seul monde ne peut pas contenir à la fois des points de vue irréductibles et une réalité objective irréductible - que l'un d'eux doit être ce qu'il y a vraiment et que l'autre doit être d'une manière ou d'une autre réductible à celui-ci ou dépendant de lui ${ }^{27}$.

Il est néanmoins possible de concevoir d'autres réponses aux problèmes métaphysiques de conflits de points de vue, intermédiaires entre l'idéalisme et le naturalisme objectiviste. Ces solutions consistent à développer des conceptions nouvelles capables d'intégrer les deux points de vue en une conception unique.

Cette voie peut être suivie de deux manières opposées: par une déflation des prétentions de l'un ou l'autre des deux points de vue ou par une élaboration métaphysique.

La voie déflationniste consiste à réinterpréter certaines des croyances portées par l'un ou l'autre des deux points de vue et à en modérer la portée de manière à ce que les conflits de points de vue se dissipent. Une solution déflationniste à l'égard du point de vue interne soutiendra ainsi par exemple que ce que le point de vue interne entend par liberté ne doit pas être interprété comme un pouvoir de création d'absolue nouveauté, comme il peut le sembler, mais comme le simple fait d'agir d'après soi-même, c'est-à-dire d'après des facteurs qui sont des propriétés du moi et non des réalités distinctes.

\section{Les solutions métaphysiques}

La voie métaphysique consiste à résoudre les conflits des points de vue en enrichissant notre conception externe de la réalité. Les solutions de ce type considèrent que les conceptions interne et externe ne sont contradictoires qu'en apparence, ou indirectement, qu'elles sont toutes les deux vraies et qu'il est nécessaire, pour surmonter leur opposition, d'élaborer une conception nouvelle de la réalité, plus riche, telle que les croyances relevant des deux points de vue cessent d'impliquer des propositions en contradiction avec l'autre point de vue. Nagel propose notamment d'adopter une position de ce type à propos du problème de la relation entre le corps et l'esprit: l'existence de la conscience, d'un "effet que cela fait ${ }^{28}$ d'être

27. T. Nagel, Mortal Questions, chap. 14, p. 212; Questions mortelles, p. 246.

28. T. Nagel, "What Is It Like to Be a Bat?», repris dans Mortal Questions, chap. 12, p. 165; Questions mortelles, p. 193. 
un certain organisme, évident du point de vue interne, et le lien très étroit entre le contenu de cette conscience et les événements cérébraux, doivent nous conduire à attribuer des propriétés nouvelles aux entités physiques décrites dans le tableau naturaliste. Ces entités, précise Nagel, consistent en des propriétés protomentales impliquant nécessairement à la fois les propriétés physiques relevées par le point de vue externe et les propriétés d'états de conscience accessibles uniquement au point de vue interne ${ }^{29}$. De manière générale, des solutions de ce type s'imposent lorsque les croyances en conflit sont si robustes qu'il n'est pas possible de les abandonner ou de les réviser suffisamment pour lever leur conflit. Dans de tels cas, nous pouvons en effet être portés à considérer que les problèmes proviennent de ce que nous cherchons à intégrer les croyances conflictuelles en jeu à l'intérieur d'une conception de la réalité qui est trop étroite et ne la reflète en vérité que partiellement. Plutôt que de réviser des croyances très solides de manière à ce que leur vérité puisse être acceptée conjointement par les deux points de vue, nous partons de la vérité de ces croyances en conflit et de l'idée que la réalité est telle que ces croyances sont conjointement vraies, et nous cherchons alors à élaborer une conception de la réalité satisfaisant cette contrainte. Nous pouvons qualifier de métaphysiques ces solutions aux problèmes posés par les conflits entre les points de vue interne et externe. En un sens large, certes, toutes les solutions réalistes aux problèmes métaphysiques sont métaphysiques, car elles acceptent le point de vue externe et affirment que la réalité s'étend au-delà du contenu immanent de notre expérience. Mais il faut distinguer entre elles des solutions métaphysiques au sens étroit, ou fort, qui résolvent les problèmes métaphysiques en postulant que la réalité comporte certains aspects dont nous ne faisons pas l'expérience, pas même indirectement, et qui échappaient tout à fait à la seconde étape de l'enquête métaphysique, la recherche d'un tableau naturaliste de la réalité. Selon ces conceptions, il faut non seulement affirmer que la réalité ne se manifeste à nous que de manière indirecte, dans notre expérience sensible, mais encore que certains aspects de la réalité ne se présentent pas même à nous de cette façon, mais seulement à travers les problèmes auxquels nous conduit le fait que nous les ignorons. La réflexion sur les problèmes de conflits entre les points de vue interne et externe constitue ici une heuristique métaphysique. Ces problèmes sont le point de départ d'une connaissance métaphysique positive, et permettent de compléter le tableau naturaliste élaboré dans le deuxième moment de la réflexion métaphysique.

29. Voir T. Nagel, The View..., partie III, p. 28-53; Le point de vue..., p. 37-66; «The Psychophysical Nexus », repris dans Concealment and Exposure, Oxford, Oxford University Press, 2002, chap. 18, p. 194-235; Mind and Cosmos..., chap. 3, p. 35-7o. 


\section{Conclusion}

Il semble possible que la réalité ne soit pas donnée entièrement à notre conscience perceptive immédiate et que celle-ci n'en soit qu'une image. Y a-t-il une réalité indépendante du contenu immédiat de notre conscience? Tel est le problème général de la métaphysique comprise au sens étroit d'une enquête à propos de ce qui dépasse l'expérience immanente du point de vue interne. La première question qui se pose à l'enquête métaphysique est cependant celle de la réalité de son objet: les idées corrélatives d'une réalité extérieure au contenu immanent et d'un point de vue externe ont-elle seulement un sens? Si nous considérons qu'elles en ont un, un deuxième problème métaphysique se pose, celui de l'élaboration d'une conception externe déterminée et adéquate. La réponse à ce problème comporte deux moments. Le premier consiste à élaborer une conception de la réalité transcendante telle qu'elle est en elle-même à partir de notre expérience. Ce premier moment est lui-même double. Il consiste d'une part en l'élaboration d'un tableau naturaliste par application de la méthode de l'objectivité, et d'autre part en une réflexion sur l'existence d'aspects de la réalité, Dieu ou la finalité naturelle par exemple, qui ne seraient pas des causes immédiates de certains aspects de notre expérience, mais pourraient se montrer dans notre expérience de manière indirecte, notamment à travers l'ordre des phénomènes, et devraient être ajoutés à la première version du tableau naturaliste, élaborée par application de la méthode de l'objectivité. La réponse au problème de la nature de la réalité transcendante comporte un second moment, car le tableau de la réalité dont nous disposons alors entre en conflit avec certaines croyances très robustes de notre conception immédiate et se trouve de ce fait ébranlé par celle-ci. Ce second moment consiste en une réflexion sur les contradictions entre notre point de vue interne immédiat et le tableau naturaliste de la réalité extérieure résultant de la réflexion sur notre expérience. La résolution des conflits entre les deux points de vue peut exiger que nous revenions sur le tableau de la réalité extérieure auquel nous sommes d'abord parvenus, et que nous lui retirions ou ajoutions certains éléments afin de faire une place satisfaisante aux intuitions les plus solides de notre point de vue interne.

Comment définir alors le travail du métaphysicien? Si l'on considère, comme nous l'avons fait, qu'il convient de déléguer aux sciences de la nature le second moment de l'enquête métaphysique, car il n'est qu'une projection de leurs résultats dans la réalité en soi, le travail qui revient en propre au métaphysicien se trouve réduit à ceci: il consiste d'une part à examiner la question de la réalité du point de vue externe, et d'autre part à résoudre les contradictions entre les points de vue interne et externe. Ce second aspect 
de la réflexion est en vérité central: en effet, nous acceptons spontanément la réalité du point de vue externe, et la question de la réalité du point de vue externe ne se pose véritablement à nous qu'en second lieu, lorsque nous constatons les contradictions entre les points de vue interne et externe et en venons à nous demander si ces problèmes ne résultent pas d'une erreur et si la négation du point de vue externe ne serait pas la meilleure manière de résoudre les contradictions auxquelles nous avons été conduits par l'acceptation du point de vue externe. Il nous est donc permis, semble-t-il, de définir la métaphysique, du moins la métaphysique comprise au sens étroit, excluant l'ontologie, comme une réflexion sur les conflits entre les points de vue interne et externe. Nagel, en isolant certains problèmes de la philosophie, en en dégageant la structure et l'origine communes et en les décrivant comme des problèmes tenant à une opposition entre ce qu'il appelle un point de vue interne et un point de vue externe, nous conduit ainsi à concevoir plus clairement le travail spécifique du métaphysicien et l'unité des tâches qui incombent à la métaphysique.

Olivier WAYMEL

\section{Références bibliographiques}

Aubenque P., Le problème de l'être chez Aristote, Paris, PUF, 1962.

Austin J. L., Sense and Sensibilia, Oxford, Oxford University Press, 1962; trad. fr.: Le langage de la perception, P. Gochet (trad.), Paris, J. Vrin, 2007.

Hamou P., Voir et connaître à l'âge classique, Paris, PUF, 2002.

Husserl E., Méditations cartésiennes: introduction à la phénoménologie, E. Levinas, G. Peiffer (trad.), Paris, J. Vrin, 1931.

Nagel T., "Dawkins and Atheism», Times Literary Supplement, 7 mai 2004; repris dans Secular Philosophy and the Religious Temperament: Essays 20022008, Oxford, Oxford University Press, 2010, p. 19-26.

Nagel T., Mind and Cosmos: Why the Materialist Neo-Darwinian Conception of Nature Is Almost Certainly False, Oxford, Oxford University Press, 2012.

Nagel T., Mortal Questions, Cambridge - Londres - New York, Cambridge University Press, 1979; trad. fr.: Questions mortelles, P. Engel, C. Tiercelin (trad.), Paris, PUF, 1983. 
Nagel T., "Public Education and Intelligent Design», Philosophy and Public Affairs, t. 36, $\mathrm{n}^{\circ}$ 2, 2008, p. 187-205; repris dans Secular Philosophy and the Religious Temperament: Essays 2002-2008, Oxford, Oxford University Press, 2010, p. 41-57.

NAgel T., The View from Nowhere, Oxford, Oxford University Press, 1986; trad. fr.: Le point de vue de nulle part, S. Kronlund (trad.), Combas, Éditions de l'éclat, 1993.

Nagel T., What Does It All Mean? A Very Short Introduction to Philosophy, Oxford, Oxford University Press, 1987; trad. fr.: Qu'est-ce que tout cela veut dire?, R. Ogien (trad.), Combas, Éditions de l'éclat, 1993.

Nagel T., «What Is It Like to Be a Bat?», Philosophical Review, t. 83, n 4, 1974, p. 435-450; repris dans Mortal Questions, Cambridge - Londres - New York, Cambridge University Press, 1979, chap. 12, p. 165-18o.

Nagel T., «Why Is There Anything?», Times Literary Supplement, 7 mai 2007; repris dans Secular Philosophy and the Religious Temperament: Essays 20022008, Oxford, Oxford University Press, 2010, p. 27-31.

Russell B., Our Knowledge of the External World as a Field for Scientific Method in Philosophy, Londres, G. Allen \& Unwin, 1922; trad. fr.: La méthode scientifique en philosophie: notre connaissance du monde extérieur, P. Devaux (trad.), Paris, Payot \& Rivages, 2002.

Strawson P. F., Skepticism and Naturalism, Londres, Routledge, 1987.

Williams B., Descartes: The Project of Pure Enquiry, Londres, Penguin Books, 1978.

Williams B., «Wittgenstein and Idealism», repris dans Moral Luck: Philosophical Papers, 1973-1980, Cambridge, Cambridge University Press, 1981, p. 144-163.

Wittgenstein L., Philosophische Untersuchungen; trad. angl.: Philosophical Investigations [I, 1936-1945; II, 1947-1949], G. E. M. Anscombe (trad.), Oxford, B. Blackwell, 1953; trad. fr.: Recherches philosophiques, F. Dastur, M. Élie, J.-L. Gautero, D. Janicaud (trad.), É. Rigal (éd.), Paris, Gallimard, 2004.

Wittgenstein L., Tractatus logico-philosophicus [1918-1921] ; éd. orig. : «Logischphilosophische Abhandlung", Annalen der Naturphilosophie, vol. XIV, $\mathrm{n}^{\circ} 3^{-4}$, 1921, p. 185-262 ; trad. fr.: Tractatus logico-philosophicus, G.-G. Granger (trad.), Paris, Gallimard, 1993. 
